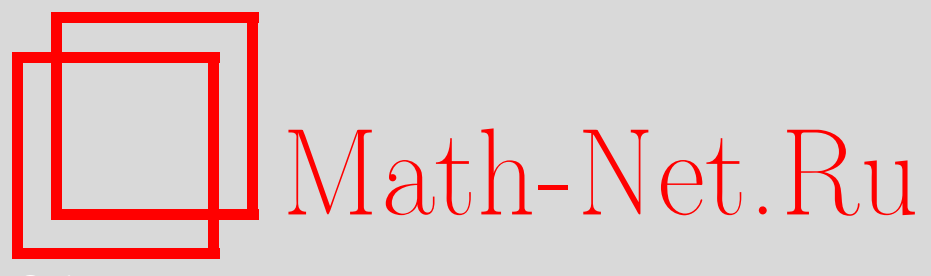

В. П. Маслов, В. Е. Назайкинский, О распределении целочисленных случайных величин, связанных двумя линейными неравенствами. I, Матем. заметки, 2008, том 83, выпуск 4, 559-580

DOI: https://doi.org/10.4213/mzm4576

Использование Общероссийского математического портала Math-Net.Ru подразумевает, что вы прочитали и согласны с пользовательским соглашением http://www . mathnet.ru/rus/agreement

Параметры загрузки:

IP : 54.172 .240 .79

26 апреля 2023 г., 08:22:46

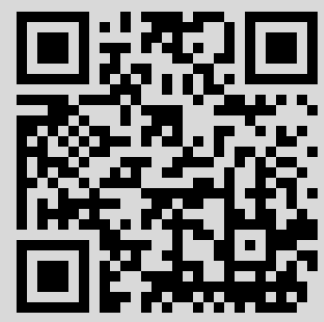


Том 83 выпуск 4 апрель 2008

УДК $519.2+531.19$

\title{
О распределении целочисленных случайных величин, связанных двумя линейными неравенствами. I
}

\author{
В. П. Маслов, В. Е. Назайкинский
}

В предыдущих статьях авторов рассматривалась задача о распределении неразличимых частиц по целым положительным уровням энергии при условии, что суммарная энергия системы ограничена сверху некоторой постоянной $M$. Были доказаны оценки, из которых следует, что при больших $M$ большинство вариантов концентрируется вблизи предельного распределения (бозе-эйнштейновского, если частицы подчиняются статистике Бозе). В данной статье мы продолжаем исследования в этом направлении и рассматриваем случай, когда задано не только ограничение на суммарную энергию, но и общее число частиц. Рассматривается как бозевское, так и гиббсовское распределение, изучается явление предельного перехода бозевского распределения в гиббсовское в случае относительно малого числа частиц.

Библиография: 4 названия.

\section{1. Введение}

В статьях [1] и [2] рассматривалась задача о распределении неразличимых частиц по целым положительным уровням энергии при условии, что суммарная энергия системы ограничена сверху некоторой постоянной $M$. Были доказаны оценки, из которых следует, что при больших $M$ большинство вариантов концентрируется вблизи предельного распределения (бозе-эйнштейновского, если частицы подчиняются статистике Бозе). В данной статье мы продолжаем исследования в этом направлении и рассматриваем случай, когда задано не только ограничение на суммарную энергию, но и общее число частиц. ${ }^{1}$ При этом оказывается, что более или менее одинаковым методом можно изучить системы как неразличимых, так и различимых частиц (в последнем случае мы естественно приходим к распределению Гиббса). Более того, если рассмотреть систему неразличимых частиц и предположить, что число частиц $N$ относительно мало (хотя и стремится к бесконечности), то из наших оценок вытекает, что бозе-эйнштейновское распределение, которому они подчиняются, становится в пределах точности, гарантируемой теоремами о концентрации, неотличимым от распределения Гиббса. Важно подчеркнуть, что коль скоро речь идет

\footnotetext{
${ }^{1} \mathrm{~B}$ частном случае, когда число частиц очень велико, это было проделано в [2], где было показано, что в этой ситуации образуется бозе-конденсат - в большинстве вариантов большинство частиц занимает самый нижний энергетический уровень, а оставшиеся частицы образуют распределение, близкое к бозе-эйнштейновскому, на остальных уровнях.
}

(С) В.П. МАСЛов, В.Е. НАЗАЙКинСКий, 2008 
о числе вариантов, это распределение не есть «настоящее» распределение Гиббса - частищы по прежнему неразличимы, и характерные для числа вариантов в распределении Гиббса факториальные множители не возникают - но доля вариантов, концентрирующихся вблизи предельной кривой, такова же, как и в «настоящем» распределении Гиббса.

В связи с тем, что мы рассматриваем системы как различимых, так и неразличимых частиц, следует более подробно остановиться на том, что́, собственно говоря, подразумевается под подсчетом вариантов распределения частиц по уровням энергии.

Пусть $\widehat{H}: \mathscr{H} \rightarrow \mathscr{H}$ - одночастичный гамильтониан, действующий в гильбертовом пространстве $\mathscr{H}$ состояний частицы. Предполагаем, что оператор $\widehat{H}$ самосопряжен, неотрицателен и имеет чисто дискретный спектр; таким образом, в $\mathscr{H}$ имеется ортонормированный базис $\left\{\psi_{j}\right\}_{j=1}^{\infty}$, состоящий из собственных функций этого оператора,

$$
\widehat{H} \psi_{j}=\lambda_{j} \psi_{j}, \quad j=1,2, \ldots
$$

(разумеется, некоторые из $\lambda_{j}$ могут совпадать между собой, т.е. оператор $\widehat{H}$ может иметь кратные собственные значения). Будем считать, что $\lambda_{j}$ упорядочены по возрастанию:

$$
\lambda_{1} \leqslant \lambda_{2} \leqslant \lambda_{3} \leqslant \cdots .
$$

Рассмотрим теперь систему $N$ невзаимодействующих частиц. Квантовомеханическое пространство состояний этой системы есть тензорное произведение

$$
\mathscr{H}_{N}=\mathscr{H}^{\otimes N} \equiv \underbrace{\mathscr{H} \otimes \mathscr{H} \otimes \cdots \otimes \mathscr{H}}_{N \text { сомножителей }}
$$

в том случае, если частицы различимы, или подпространство $\mathscr{H}_{N}^{B}$ этого тензорного произведения, состоящее из симметрических тензоров, в случае, если частицы неразличимы и подчиняются статистике Бозе-Эйнштейна.

Гамильтониан $\widehat{H}_{N}$ рассматриваемой системы имеет вид

$$
\widehat{H}_{N}=\underbrace{\widehat{H} \otimes 1 \otimes \cdots \otimes 1+1 \otimes \widehat{H} \otimes 1 \otimes \cdots \otimes 1+1 \otimes \cdots \otimes 1 \otimes \widehat{H}}_{N \text { слагаемых }} .
$$

(Это выражение годится как для различимых, так и для неразличимых частиц, ибо подпространство $\mathscr{H}_{N}^{B}$ инвариантно относительно $\widehat{H}_{N}$.)

В пространстве $\mathscr{H}_{N}$ есть ортонормированный базис, состоящий из элементов вида

$$
\Psi_{j_{1}, \ldots, j_{N}}=\psi_{j_{1}} \otimes \psi_{j_{2}} \otimes \cdots \otimes \psi_{j_{N}} .
$$

В бозонном случае базис в $\mathscr{H}_{N}^{B}$ образуют симметрические элементы

$$
\Psi_{j_{1}, \ldots, j_{N}}=\sum_{\sigma \in \mathfrak{S}_{N}} \psi_{j_{\sigma}(1)} \otimes \psi_{j_{\sigma}(2)} \otimes \cdots \otimes \psi_{j_{\sigma}(N)}
$$

(сумма в (2) берется по всем перестановкам $N$ элементов). 
Функция (1) (соответственно (2)) является собственной функцией $N$-частичного гамильтониана $\widehat{H}_{N}$ в пространстве $\mathscr{H}_{N}$ (соответственно $\mathscr{H}_{N}^{B}$ ) с собственным значением

$$
\lambda \equiv \lambda_{j_{1}, \ldots, j_{N}}=\lambda_{j_{1}}+\cdots+\lambda_{j_{N}} .
$$

Различные функции описывают различные собственные состояния гамильтониана $\widehat{H}_{N}$, и когда речь идет о подсчете числа вариантов распределения частиц по уровням энергии, это равносильно подсчету числа этих собственных состояний, или, что то же самое, подсчету числа собственных значений гамильтониана $\widehat{H}_{N}$ с учетом кратности.

На языке математической статистики это можно сформулировать следующим образом: каждое конкретное распределение $N$ частиц по уровням энергии есть не что иное, как въборка объема $N$ (см., например, [4]) из множества уровней энергии. Для случая различимых частиц это упорядоченная выборка (существенно, какая именно частица попала на какой уровень), а для случая неразличимых частиц - выборка неупорядоченная. Более того, в данной работе мы рассматриваем только въборки c возвращениями - число частиц на одном и том же уровне может быть произвольным. (Если же рассматривать частицы, подчиняющиеся ферми-статистике, когда на одном и том же уровне энергии не может находиться более одной частицы, то следует использовать выборки без возвращения - однажды «извлеченный» уровень энергии в дальнейших попытках не участвует. Впрочем, в данной работе эта задача не затрагивается.)

Итак, рассматриваемые нами распределения частиц по уровням энергии суть выборки $\left\{j_{1}, \ldots, j_{N}\right\}$ из множества уровней, а именно,

- упорядоченные выборки с возвращением для случая различимых частиц,

- неупорядоченные выборки с возвращением для случая неразличимых частиц.

Даже если частицы различимы, в каждой конкретной выборке нас будет интересовать лишь число частиц, находящихся на каждом из уровней. Обозначим через $N_{j}$ число раз, которое сомножитель $\psi_{j}$ встречается в произведении в правой части формулы (1) или (2) (т.е. число тех $s \in\{1, \ldots, N\}$, для которых $j_{s}=j$ ). Вектор $\Psi_{j_{1}, \ldots, j_{N}}$ (или, что то же самое, выборка $\left\{j_{1}, \ldots, j_{N}\right\}$ ) отвечает конкретному квантовомеханическому состоянию системы из $N$ частиц а число $N_{j}$ показывает, сколько именно из этих частиц находится в $j$-м состоянии $\psi_{j}$. Числа $N_{j}$ называются числами заполнения. Итак, каждой выборке $\left\{j_{1}, \ldots, j_{N}\right\}$ (т.е. каждому собственному вектору (1) или (2) $N$-частичного гамильтониана $\left.\widehat{H}_{N}\right)$ сопоставляется набор $\left(N_{1}, N_{2}, \ldots\right)$ чисел заполнения, причем, очевидно,

$$
N_{1}+N_{2}+\cdots=N, \quad \lambda_{1} N_{1}+\lambda_{2} N_{2}+\cdots=\lambda .
$$

Не представляет также труда для заданного собственного вектора (1) или (2) определить число заполнения, отвечающее интервалу энергий $\left[E_{1}, E_{2}\right]$ одночастичного гамильтониана. Именно,

$$
N_{E_{1}, E_{2}}=N_{j}+N_{j+1}+\cdots+N_{j+s}
$$

где $\lambda_{j}, \lambda_{j+1}, \ldots, \lambda_{j+s}$ - все уровни энергии одночастичного гамильтониана на отрезке $\left[E_{1}, E_{2}\right]$. 
ЗАмечАниЕ 1. В бозонном случае (т.е. для неупорядоченной выборки) набор чисел заполнения однозначно определяет выборку, в то время как в «гиббсовском» случае одному и тому же набору чисел заполнения отвечает $N ! /\left(N_{1} ! N_{2} ! \cdots\right)$ различных упорядоченных выборок, отличающихся друг от друга перестановками.

Поэтому при подсчете выборок в бозонном случае можно просто считать всевозможные наборы чисел заполнения. В гиббсовском случае, чтобы подсчитать выборки, также можно считать наборы чисел заполнения, но уже с весами (коэффициентами) $N ! /\left(N_{1} ! N_{2} ! \cdots\right)$.

ЗАмечАниЕ 2. Ничто не препятствует тому, чтобы рассмотреть упорядоченные выборки без возвращения (при этом получится описание системы различимых частиц, подчиняющихся принципу запрета Паули). Насколько нам известно, такие системы в литературе не рассматривались.

ЗАмЕчАниЕ 3. К ограничению на энергию мы добавили еще одно - условие-равенство для числа частиц. По крайней мере в случае бозе-частиц можно было бы и на число частиц наложить условие типа неравенства. При этом если $M$ есть ограничение на энергию системы, то существует такое $N_{0}=N_{0}(M)$, что при $N \gg N_{0}$ условие, что число частиц не превосходит $N$, приводит к таким же результатам, что и отсутствие какого бы то ни было условия на число частиц, а при $N<N_{0}$ условие-неравенство и условие-равенство приводят к качественно одинаковым результатам.

\section{2. Распределение Бозе-Эйнштейна для целочисленных уровней энергии}

Рассмотрим задачу о распределении тождественных неразличимых частиц по целочисленным уровням энергии при условии, что суммарная энергия системы ограничена сверху, а число частиц задано. Частицы будем считать бозевскими (т.е. на каждом уровне энергии может находиться неограниченное количество частиц). Для простоты будем рассматривать систему размерности $d=3$.

2.1. Определение системы. Будем изучать систему, определяемую следующим образом. Для уровней энергии $j=0,1,2, \ldots$ кратностей

$$
q_{j}=\frac{(j+1)(j+2)}{2}, \quad j=0,1,2, \ldots,
$$

рассматриваются всевозможные наборы $\left\{N_{j k}\right\}$ неотрицательных целых чисел $N_{j k}$, $j=0,1,2, \ldots, k=1, \ldots, q_{j}$, удовлетворяющих условиям

$$
\begin{gathered}
\sum_{j=0}^{\infty} N_{j k}=N \\
\sum_{j=0}^{\infty} \sum_{k=1}^{q_{j}} j N_{j k} \equiv \sum_{j=1}^{\infty} \sum_{k=1}^{q_{j}} j N_{j k} \leqslant M,
\end{gathered}
$$

где $N$ и $M$ - заданные положительные числа (которые без ограничения общности будем считать целыми). Все такие наборы будем считать равновероятными. 
ЗАмЕчАниЕ 4. В работе [1] значения уровней энергии начинались не с нуля, а с единицы - иначе при отсутствии ограничений на число частиц общее число вариантов оказалось бы бесконечным. В присутствии ограничения (5) удобнее рассматривать уровни энергии, начинающиеся с нуля - формулы при этом упрощаются. При желании, разумеется, можно перейти и к уровням, начинающимся с единицы, сделав замену

$$
M \mapsto M+N, \quad q_{j} \mapsto q_{j-1} .
$$

Нас интересует, что будет происходить с системой при $M \rightarrow \infty$ и $N \rightarrow \infty$. Введем дополнительные числовые характеристики системы, зависящие $M$ и $N$, в терминах которых и будут формулироваться результаты.

Определим числа $b=b(M, N)>0$ и $z=z(M, N)>1$ как решение системы уравнений

$$
M=\sum_{j=1}^{\infty} \frac{j q_{j}}{z e^{b j}-1}, \quad N=\sum_{j=0}^{\infty} \frac{q_{j}}{z e^{b j}-1} .
$$

ПреДЛОЖЕНИЕ 5. Для любых $M, N>0$ система (8) имеет единственное решение $b=b(M, N)>0, z=z(M, N)>1$. Далее, при $b \rightarrow 0$ справедливы асимптотические соотношения

$$
N \asymp z^{-1} b^{-3} \quad \text { npu } z>1+b^{3}, \quad M \asymp z^{-1} b^{-4} .
$$

ЗАмечАниЕ 6. Как и в [1], можно в системе (8) перейти от сумм к интегралам, пользуясь формулами Эйлера-Маклорена. В главном члене при этом получим (для простоты ограничиваясь случаем, когда $z-1$ не мало́) [3; с. 339, 3.411-6]

$$
N=z^{-1} b^{-3}\left({ }_{1} F_{1}\left(z^{-1} ; 3 ; 1\right)+O(b)\right), \quad M=3 z^{-1} b^{-4}\left({ }_{1} F_{1}\left(z^{-1} ; 4 ; 1\right)+O(b)\right),
$$

где ${ }_{1} F_{1}(a ; b ; c)$ - вырожденная гипергеометрическая функция первого рода.

В дальнейшем, если явно не оговорено противное, всюду считается, что $M, N, b$ и $z$ связаны формулами (8), а параметры $z$ и $b$ удовлетворяют соотношениям

$$
1+b^{3 / 2-\varepsilon}<z<b^{-3+\varepsilon}
$$

для некоторого произвольно зафиксированного $\varepsilon>0$.

Кроме того, мы введем обозначения

$$
N_{j}=\sum_{k=0}^{q_{j}} N_{j k}
$$

для суммарных чисел заполнения соответствующих уровней $j$ и положим

$$
\bar{N}_{j}=\frac{q_{j}}{z e^{b j}-1}, \quad j=0,1,2, \ldots
$$

2.2. Энтропия и теоремы о концентрации. Обозначим через $\mathscr{N}(M, N)$ общее число наборов $\left\{N_{j k}\right\}$, удовлетворяющих ограничениям (5), (6).

ОПРЕДЕЛЕНИЕ 7. Энтропией рассматриваемой системы назовем число

$$
S=\ln \mathscr{N}(M, N)
$$


Асимптотику энтропии при $N, M \rightarrow \infty$ дает следующая теорема.

Теорема 8. Пусть выполнено соотношение (10). Тогда при $N, M \rightarrow \infty$ справедлива асимптотическая формула

$$
S=N \ln z+b M+\sum_{j=1}^{\infty} q_{j} \ln \frac{1}{1-z^{-1} e^{-b j}}+O(\ln N) .
$$

Пусть $\psi(x)$ - фиксированная положительная функция, которая стремится к $+\infty$ при $x \rightarrow+\infty$, и пусть

$$
\Delta N=\sqrt{N \ln N} \psi(N), \quad \Delta M=\frac{M}{N} \Delta N .
$$

Теорема 9. Пусть выполнено соотношение (10). Тогда

$$
\mathrm{P}\left(\left|\sum_{j=0}^{\infty} j N_{j}-M\right|>\Delta M\right)=O\left(N^{-k}\right)
$$

для любого $k$.

Для кумулятивных вероятностей справедлива следующая

Теорема 10. Пусть выполнено соотношение (10). Тогда для любого $l \in \mathbb{Z}_{+}$ справедливы оценки

$$
\begin{gathered}
\mathrm{P}\left(\left|\sum_{j=0}^{l} N_{j}-\sum_{j=1}^{l} \frac{q_{j}}{z e^{b j}-1}\right|>\Delta N\right)=O\left(N^{-k}\right), \\
\mathrm{P}\left(\left|\sum_{j=1}^{l} j N_{j}-\sum_{j=1}^{l} \frac{j q_{j}}{z e^{b j}-1}\right|>\Delta M\right)=O\left(N^{-k}\right)
\end{gathered}
$$

для любого $k$.

2.3. Доказательства. Доказательство утверждений из предыдущего пункта, как и в [1], разобьем на несколько подпунктов.

2.3.1. Оценка сверху для общего числа вариантов. Обозначим

$$
\mu=\ln z, \quad \text { так что } \quad z=e^{\mu} .
$$

Пусть $\mathscr{M}$ - множество всех выборок, на котором выполнены условия (5) и (6). Из этих условий вытекает, что на множестве $\mathscr{M}$ для соответствующих наборов $\left\{N_{j k}\right\}$ чисел заполнения справедливо неравенство

$$
\exp \left\{b M+\mu N-\sum_{j=0}^{\infty} \sum_{k=1}^{q_{j}} N_{j k}(b j+\mu)\right\} \geqslant 1 .
$$

(Это неравенство справедливо для любых положительных $b$ и $\mu$, но мы будем использовать именно $b$ и $\mu$, определенные выше.) Суммируя это неравенство сначала 
по всем наборам $\left\{N_{j k}\right\}$ из $\mathscr{M}$, а затем распространяя суммирование на всевозможные финитные неотрицательные $\left\{N_{j k}\right\}$, получаем

$$
\begin{aligned}
\mathscr{N}(M, N) & =\sum_{\left\{N_{j k}\right\} \in \mathscr{M}} 1 \leqslant e^{b M+\mu N} \sum_{\left\{N_{j k}\right\}} \exp \left\{-\sum_{j=0}^{\infty} \sum_{k=1}^{q_{j}} N_{j k}(b j+\mu)\right\} \\
& =e^{b M+\mu N} \prod_{j=0}^{\infty} \prod_{k=1}^{q_{j}} \sum_{N_{j k}=0}^{\infty} e^{-N_{j k}(b j+\mu)} \\
& =e^{b M+\mu N} \prod_{j=0}^{\infty} \prod_{k=1}^{q_{j}} \frac{1}{1-e^{-b j-\mu}} \\
& =\exp \left\{b M+\mu N+\sum_{j=0}^{\infty} q_{j} \ln \frac{1}{1-e^{-b j-\mu}}\right\} .
\end{aligned}
$$

Это и есть искомая оценка сверху для числа вариантов.

2.3.2. Оценка числа вариантов с большими уклонениями. Оценим теперь сверху число вариантов из $\mathscr{M}$, для которых

$$
\left|\sum_{j=0}^{l}\left(N_{j}-\bar{N}_{j}\right)\right|>\Delta
$$

где $\bar{N}_{j}=q_{j}\left(z e^{b j}-1\right)^{-1}-$ значения (12) чисел заполнения, отвечающие бозевскому распределению. Множество таких вариантов обозначим через $\mathscr{M}_{\Delta}$.

Положим для удобства записи дальнейших формул

$$
\bar{N}_{j k}=\frac{1}{z e^{b j}-1} \equiv \bar{N}_{j} / q_{j}
$$

(эти числа не зависят от $k$ ). На множестве $\mathscr{M}_{\Delta}$ выполнены неравенства $(5),(6)$ и

$$
\Delta-\sum_{j=0}^{l} \sum_{k=1}^{q_{j}}\left(N_{j k}-\bar{N}_{j k}\right) \leqslant 0 \quad \text { или } \quad \Delta-\sum_{j=0}^{l} \sum_{k=1}^{q_{j}}\left(\bar{N}_{j k}-N_{j k}\right) \leqslant 0 .
$$

Поэтому на этом множестве справедливо и неравенство

$$
\begin{aligned}
\exp \{b M & \left.+\mu N-\sum_{j=0}^{\infty} \sum_{k=1}^{q_{j}} N_{j k}(b j+\mu)\right\} \\
& \times\left(\exp \left\{c \sum_{j=0}^{l} \sum_{k=1}^{q_{j}}\left(N_{j k}-\bar{N}_{j k}\right)\right\}+\exp \left\{c \sum_{j=0}^{l} \sum_{k=1}^{q_{j}}\left(\bar{N}_{j k}-N_{j k}\right)\right\}\right) \geqslant 1 .
\end{aligned}
$$


Пусть $\mathscr{N}(M, N, \Delta)$ - число вариантов во множестве $\mathscr{M}_{\Delta}$. Рассуждая как и выше при оценке величины $\mathscr{N}(M, N)$, получаем

$$
\begin{aligned}
\mathscr{N}(M, N, \Delta) \leqslant \exp \{b & \left.M+\mu N-c \Delta+\sum_{j=l+1}^{\infty} q_{j} \ln \frac{1}{1-e^{-b j-\mu}}\right\} \\
\times & \left(\exp \left\{\sum_{j=0}^{l}\left(q_{j} \ln \frac{1}{1-e^{-b j-\mu+c}}-c \bar{N}_{j}\right)\right\}\right. \\
+ & \left.\exp \left\{\sum_{j=0}^{l}\left(q_{j} \ln \frac{1}{1-e^{-b j-\mu-c}}+c \bar{N}_{j}\right)\right\}\right) .
\end{aligned}
$$

Эта формула справедлива при $0<c<\mu$. Воспользуемся формулой Тейлора с остаточным членом второго порядка и получим

$$
\begin{aligned}
q_{j} \ln \frac{1}{1-e^{-b j-\mu+c}}-c \bar{N}_{j}= & q_{j} \ln \frac{1}{1-e^{-b j-\mu}}+c\left(\frac{q_{j}}{e^{b j+\mu}-1}-\bar{N}_{j}\right) \\
& +\frac{q_{j} c^{2} e^{b j+\mu-\theta_{j} c}}{\left(e^{b j+\mu-\theta_{j} c}-1\right)^{2}} \\
= & q_{j} \ln \frac{1}{1-e^{-b j-\mu}+\frac{q_{j} c^{2} e^{b j+\mu-\theta_{j} c}}{\left(e^{b j+\mu-\theta_{j} c}-1\right)^{2}},}
\end{aligned}
$$

где $\theta_{j} \in[0,1]$, и аналогично для второго слагаемого в $(25)$. Если $c \leqslant \min \{\mu / 2,1\}$, то, рассуждая, как и в [1], с использованием оценок, аналогичных полученным там в лемме 23, получим неравенство

$$
\sum_{j=0}^{l} \frac{q_{j} e^{b j+\mu \pm \theta_{j} c}}{\left(e^{b j+\mu \pm \theta_{j} c}-1\right)^{2}} \leqslant \text { const } e^{-\mu} b^{-3}
$$

Следовательно,

$$
\mathscr{N}(N, M, \Delta) \leqslant \exp \left\{b M+\mu N+\sum_{j=0}^{\infty} q_{j} \ln \frac{1}{1-e^{-b j-\mu}}\right\} \exp \left\{-c \Delta+K c^{2} e^{-\mu} b^{-3}\right\} .
$$

Подставляя сюда

$$
\Delta=\Delta N=\sqrt{N \ln N} \psi(N) \asymp e^{-\mu / 2} b^{-3 / 2} \sqrt{\ln N} \psi(N)
$$

И

$$
c=\frac{b^{3} e^{\mu} \Delta}{2 K} \asymp b^{3 / 2} e^{\mu / 2} \sqrt{\ln N} \psi(N)
$$

(что законно, поскольку при таком выборе $c$ в силу условия (10) неравенство $c \leqslant \mu / 2$ будет заведомо выполнено при достаточно больших $N$ ), получим неравенство

$$
\begin{aligned}
\mathscr{N}(N, M, \Delta) & \leqslant \exp \left\{b M+\mu N+\sum_{j=0}^{\infty} q_{j} \ln \frac{1}{1-e^{-b j-\mu}}\right\} N^{-\psi^{2}(N) / 2} \\
& \leqslant C_{k} \exp \left\{b M+\mu N+\sum_{j=0}^{\infty} q_{j} \ln \frac{1}{1-e^{-b j-\mu}}\right\} N^{-k}
\end{aligned}
$$

для любого $k$. Это и есть требуемая оценка сверху для числа вариантов с большими уклонениями. 
2.3.3. Оценка снизу для общего числа вариантов. Дадим теперь для $\mathscr{N}(M, N)$ оценку снизу. Вместо того, что бы оценивать само число $\mathscr{N}(M, N)$, оценим число $\mathscr{N}_{0}(M, N)<\mathscr{N}(M, N)$ вариантов, для которых выполнены соотношения (5) и (6), причем последнее также обращается в равенство,

$$
\sum_{j=0}^{\infty} \sum_{k=1}^{q_{j}} j N_{j k}=M
$$

Аналогично тому, как это было проделано в работе [1] (формулы (61)-(64)), используя интегральное представление

$$
\delta_{m n}=\frac{b}{2 \pi} \int_{-\pi / b}^{\pi / b} e^{i b \varphi(m-n)} d \varphi
$$

символа Кронекера, запишем $\mathscr{N}_{0}(M, N)$ в виде интеграла

$$
\mathscr{N}_{0}(M, N)=\frac{b}{4 \pi^{2}} e^{b M+\mu N} \int_{-\pi / b}^{\pi / b}\left(\int_{-\pi}^{\pi} e^{\Lambda \Phi(\varphi, \psi)} d \psi\right) d \varphi,
$$

где

$$
\begin{gathered}
\Lambda=b^{-3} e^{-\mu} \asymp N \\
\Phi(\varphi, \psi)=i b^{4} e^{\mu} M \varphi+i b^{3} e^{\mu} N \psi+b^{3} e^{\mu} \sum_{j=0}^{\infty} q_{j} \ln \frac{1}{1-e^{-\mu-b j-i(\psi+b j \varphi)}} .
\end{gathered}
$$

ЛЕмма 11. Задаваемая формулой (34) фазовая функиия $\Phi(\varphi, \psi)$ обладает следующими свойствами:

1) все ее производные равномерно ограничены при значениях параметров $b u z$, удовлетворяющих неравенствам (10);

2) фазовая функиия имеет стационарную точку $\varphi=0 \bmod 2 \pi / b, \psi=0 \bmod 2 \pi$;

3) матрица $\Phi^{\prime \prime}(0,0)$ вторых производных фазовой функции в стационарной точке невырождена и равномерно по параметрам $b$ и z, удовлетворяющим неравенствам (10), отрицательно определена;

4) мнимая часть фазовой функции в стационарной точке равна нулю, а ее вещественная часть достигает там абсолютного максимума, причем для любого $\gamma>0$ существует такое $\delta>0$, не зависящее от параметров $b u z$, удовлетворяющих неравенствам (10), что

$$
\operatorname{Re} \Phi(\varphi, \psi)<\operatorname{Re} \Phi(0,0)-\delta \quad n p u \quad \operatorname{dist}((\varphi, \psi),(0,0))>\gamma
$$

ДокАЗАТЕЛЬСтво. Доказательство этой леммы вполне аналогично доказательству леммы 18 из [1], с некоторыми отличиями, вызванными тем фактом, что здесь, в отличие от [1], рассматривается двумерный, а не одномерный интеграл. Поэтому мы лишь отметим основные моменты доказательства.

1) Ограниченность первых и вторых производных фазовой функции доказывается непосредственной выкладкой. Ограниченность дальнейших производных доказывается тем же методом, что и в [1], индукцией по порядку производной. 
2) Чтобы проверить, что точка $(0,0)$ - стационарная точка фазовой функции, вычислим ее первые производные:

$$
\begin{aligned}
& \frac{\partial \Phi}{\partial \varphi}=i b^{4} e^{\mu}\left[M-\sum_{j=0}^{\infty} \frac{j q_{j}}{e^{\mu+b j+i(\psi+b j \varphi)}-1}\right] \\
& \frac{\partial \Phi}{\partial \psi}=i b^{3} e^{\mu}\left[N-\sum_{j=0}^{\infty} \frac{q_{j}}{e^{\mu+b j+i(\psi+b j \varphi)}-1}\right] .
\end{aligned}
$$

При $\varphi=\psi=0$ обе производные обращаются в нуль в силу определения параметров $b$ и $z=e^{\mu}$.

3) Матрица $\Phi^{\prime \prime}(0,0)$ имеет вид

$$
\Phi^{\prime \prime}(0,0)=-\sum_{j=0}^{\infty} \frac{b^{3} q_{j} e^{b j}}{\left(e^{b j}-e^{-\mu}\right)^{2}}\left(\begin{array}{cc}
1 & b j \\
b j & b^{2} j^{2}
\end{array}\right) .
$$

В этой формуле все слагаемые (рассматриваемые как матрицы квадратичных форм) неположительно определены, и мы можем, опуская часть слагаемых и выбрасывая $e^{-\mu}$ в знаменателе, оценить эту матрицу следующим образом:

$$
\Phi^{\prime \prime}(0,0) \leqslant-\sum_{j=\left[x_{1} / b\right]}^{\left[x_{2} / b\right]} b^{3} q_{j} e^{-b j}\left(\begin{array}{cc}
1 & b j \\
b j & b^{2} j^{2}
\end{array}\right),
$$

где $x_{2}>x_{1}>0$ - произвольные фиксированные числа. При малых $b$ матрицу в правой части можно с учетом асимптотики $q_{j} \simeq j^{2} / 2$ при больших $j$ вычислить по формуле Эйлера-Маклорена, получая в результате (с точностью до o(1)) матрицу

$$
-\frac{1}{2}\left(\begin{array}{ll}
\int_{x_{1}}^{x_{2}} x^{2} e^{-x} d x & \int_{x_{1}}^{x_{2}} x^{3} e^{-x} d x \\
\int_{x_{1}}^{x_{2}} x^{3} e^{-x} d x & \int_{x_{1}}^{x_{2}} x^{4} e^{-x} d x
\end{array}\right)=-\frac{1}{2}\left(\begin{array}{ll}
(1,1) & (1, x) \\
(x, 1) & (x, x)
\end{array}\right),
$$

где

$$
(u, v)=\int_{x_{1}}^{x_{2}} u(x) \bar{v}(x) e^{-x} x^{2} d x
$$

- скалярное произведение в $L^{2}\left(\left[x_{1}, x_{2}\right], e^{-x} x^{2}\right)$. Поскольку функции 1 и $x$ линейно независимы, матрица (38) отрицательно определена, что и доказывает требуемое утверждение.

4) Справедливо соотношение

$$
\operatorname{Re} \Phi(0,0)-\operatorname{Re} \Phi(\varphi, \psi)=b^{2} \sum_{j=0}^{\infty} q_{j} \Delta_{j}
$$

где

$$
\Delta_{j}=z \ln \frac{1}{1-z^{-1} e^{-b j}}-z \ln \left|\frac{1}{1-z^{-1} e^{-b j(1+i \varphi)+i \psi}}\right|
$$


Преобразуя это выражение подобно тому, как это было сделано в [1; с.246-247], получаем

$$
\begin{aligned}
\operatorname{Re} \Phi(0,0)-\operatorname{Re} \Phi(\varphi, \psi) & \geqslant \operatorname{const} b \sum_{j=\left[x_{1} / b\right]}^{\left[x_{2} / b\right]}(1-\cos (b j \varphi+\psi)) \\
& \geqslant \operatorname{const}\left(x_{2}-x_{1}-b-b\left|\sin \frac{b \varphi}{2}\right|^{-1}\right)
\end{aligned}
$$

(мы воспользовались формулой [3; 1.341 .3$]$ для суммы косинусов) откуда, подбирая $x_{1}$ и $x_{2}$ подходящим образом, получаем требуемое утверждение. Лемма доказана.

Теперь мы можем оценить интеграл (32). По лемме 11 все производные функции $\Phi(\varphi, \psi)$ равномерно ограничены. Кроме того, если представить ее в виде $\Phi=$ $\Phi_{1}+i \Phi_{2}$, где $\Phi_{1}$ и $\Phi_{2}$ вещественны, то

$$
\operatorname{grad} \Phi_{1}(0,0)=0, \quad \Phi_{1}^{\prime \prime}(0,0)<-C<0, \quad \Phi_{2}^{\prime}(0,0)=0, \quad \Phi_{2}^{\prime \prime}(0,0)=0 .
$$

Отсюда с помощью формулы Тейлора с остаточным членом получаем оценки

$$
\begin{gathered}
\Phi(0,0)-C_{1}\left(\varphi^{2}+\psi^{2}\right) \leqslant \Phi_{1}(\varphi, \psi) \leqslant \Phi(0,0)-C_{2}\left(\varphi^{2}+\psi^{2}\right), \\
\left|\Phi_{2}(\varphi)\right| \leqslant C_{3}\left(\varphi^{2}+\psi^{2}\right)^{3 / 2}
\end{gathered}
$$

с положительными постоянными $C_{j}$, не зависящими от параметров $b$ и $z$, при условии, что $\sqrt{\varphi^{2}+\psi^{2}} \leqslant \varepsilon$, где $\varepsilon>0$ достаточно мало. Пусть

$$
1=\chi_{1}(\varphi, \psi)+\chi_{2}(\varphi, \psi)
$$

- неотрицательное гладкое разбиение единицы на торе

$$
T^{2} \ni(\varphi \bmod 2 \pi / b, \psi \bmod 2 \pi)
$$

такое, что

$$
\chi_{1}(\varphi, \psi)= \begin{cases}0 & \text { при } \sqrt{\varphi^{2}+\psi^{2}} \geqslant \varepsilon \\ 1 & \text { при } \sqrt{\varphi^{2}+\psi^{2}} \leqslant \varepsilon / 2\end{cases}
$$

Представим интеграл

$$
I=\iint_{T^{2}} e^{\Lambda \Phi(\varphi, \psi)} d \varphi d \psi
$$

в виде суммы

$$
I=\iint_{T^{2}} e^{\Lambda \Phi(\varphi, \psi)} \chi_{1}(\varphi, \psi) d \varphi d \psi+\iint_{T^{2}} e^{\Lambda \Phi(\varphi, \psi)} \chi_{2}(\varphi, \psi) d \varphi d \psi \equiv I_{1}+I_{2} .
$$

По лемме 11, п. 4

$$
\operatorname{Re} \Phi(\varphi, \psi) \leqslant \Phi(0,0)-\delta, \quad \delta>0,
$$

на носителе подынтегрального выражения в $I_{2}$, а мера носителя имеет порядок $b^{-1}$. Поэтому

$$
\left|I_{2}\right| \leqslant K e^{\Lambda(\Phi(0,0)-\delta / 2)}
$$


с некоторой постоянной $K$. Оценим теперь интеграл $I_{1}$. Для этого рассмотрим диск

$$
D=D(0, \varepsilon)
$$

радиуса $\varepsilon$ с центром в точке $(0,0)$ и в нем два меньших диска $D_{1 / 2} \subset D_{1 / 3} \subset D$, где

$$
D_{1 / 2}=D\left(0, \varepsilon \Lambda^{-1 / 2}\right), \quad D_{1 / 3}=D\left(0, \varepsilon \Lambda^{-1 / 3}\right)
$$

Тогда

$$
\left|\Lambda \Phi_{2}\right| \leqslant C_{3} \varepsilon^{3} \quad \text { при } \quad \varphi \in D_{1 / 3},
$$

так что (при достаточно малом $\varepsilon$ ) в диске $D_{1 / 3}$ мнимая часть аргумента экспоненты мала и справедливо соотношение

$$
\operatorname{Re} e^{\Lambda \Phi(\varphi, \psi)} \geqslant \frac{1}{2} e^{\Lambda \Phi_{1}(\varphi, \psi)}, \quad \varphi \in D_{1 / 3} .
$$

Далее,

$$
\Lambda \Phi(0,0) \geqslant \Lambda \Phi_{1}(\varphi, \psi) \geqslant \Lambda \Phi(0,0)-C_{1} \varepsilon^{2}, \quad \varphi \in D_{1 / 2}
$$

Комбинируя это с предыдущим неравенством и учитывая, что площадь диска $D_{1 / 2}$ равна $\pi \varepsilon^{2} \Lambda^{-1}$, получаем

$$
\operatorname{Re} \iint_{D_{1 / 2}} e^{\Lambda \Phi(\varphi, \psi)} \psi_{1}(\varphi, \psi) d \varphi d \psi \geqslant C_{4} e^{\Lambda \Phi(0,0)} \Lambda^{-1}
$$

Далее,

$$
\operatorname{Re} \iint_{D_{1 / 3} \backslash D_{1 / 2}} e^{\Lambda \Phi(\varphi, \psi)} \psi_{1}(\varphi, \psi) d \varphi d \psi \geqslant 0
$$

в силу (44). Кроме этого, справедливо неравенство

$$
\Lambda \Phi_{1}(\varphi, \psi) \leqslant \Lambda \Phi_{1}(0,0)-C_{2} \varepsilon^{2} \Lambda^{1 / 3}, \quad(\varphi, \psi) \in D \backslash D_{1 / 3},
$$

так что

$$
\left|\iint_{D \backslash D_{1 / 3}} e^{\Lambda \Phi(\varphi, \psi)} \psi_{1}(\varphi, \psi) d \varphi d \psi\right| \leqslant C_{5} e^{\Lambda \Phi}(0,0) e^{-C_{2} \varepsilon^{2} \Lambda^{1 / 3}} .
$$

Комбинируя все предыдущие оценки, получаем

$$
I=\operatorname{Re} I \geqslant C_{6} \Lambda^{-1} e^{\Lambda \Phi(0,0)} .
$$

Остается подставить эту оценку в формулу для $\mathscr{N}_{0}(M, N)$ с учетом выражения для фазовой функции, и в силу неравенства $\mathscr{N}(M, N)>\mathscr{N}_{0}(M, N)$ мы приходим к следующей оценке снизу для $\mathscr{N}(M, N)$ (ср. рассуждения, проведенные в [1]):

$$
\mathscr{N}(N, M) \geqslant C b \Lambda^{-1} \exp \left\{b M+\mu N+\sum_{j=0}^{\infty} q_{j} \ln \frac{1}{1-e^{-b j-\mu}}\right\} .
$$


2.3.4. Вычисление энтропии и доказательство теорем о концентрации. Теперь несложно доказать теоремы 8-10. Именно, теорема 8 (асимптотика энтропии) получается, если скомбинировать верхнюю и нижнюю оценки $(20)$ и $(46)$ для $\mathscr{N}(N, M)$ и перейти к логарифмам. Неравенство (17) получается из оценок (31) и (46). Чтобы доказать неравенство (18), для соответствующего числа вариантов с большими уклонениями мы можем вывести оценку, аналогичную (31) (рассуждения тут аналогичны проведенным выше, а похожее вычисление читатель может найти в [1]) и скомбинировать ее с (46). Тем самым получается теорема 10. Наконец, теорема 9 является частным случаем теоремы 10 (при $l=\infty$ ).

\section{3. Распределение Гиббса для целочисленных уровней энергии}

Рассмотрим теперь задачу о распределении тождественных частиц по целым уровням энергии с ограничениями на суммарную энергию и число частиц, считая, что частицы различимы. Мы по-прежнему считаем, что на каждом уровне энергии может находиться неограниченное количество частиц и рассматриваем систему размерности $d=3$.

3.1. Определение системы. По сравнению со случаем неразличимых частиц определение системы теперь претерпевает некоторые изменения. Именно, как уже отмечалось выше, для различимых частиц каждому набору чисел заполнения соответствует целое множество выборок, и поэтому в качестве равноправных вариантов в этом случае следует брать не наборы чисел заполнения, а сами выборки. Итак, пусть $\mathscr{E}-$ множество уровней энергии $j=0,1,2, \ldots$ кратностей $q_{j}$, задаваемых формулой (4). Рассмотрим всевозможные упорядоченные выборки с возвращениями из этого множества, такие, что соответствующие этим выборкам наборы $\left\{N_{j k}\right\}$, $j=0,1,2, \ldots, k=1, \ldots, q_{j}$, чисел заполнения удовлетворяют условиям (5) и (6), где $N$ и $M$ - заданные целые положительные числа. (Первое из этих условий означает просто, что объем выборки равен $N$, а второе - что энергия отвечающего этой выборке состояния $N$-частичной системы не превосходит $M$.) Нас интересует, что будет происходить с системой при $M \rightarrow \infty$ и $N \rightarrow \infty$.

Как и в случае различимых частиц, введем дополнительные числовые характеристики системы, в терминах которых будут формулироваться результаты.

Определим числа $\beta=\beta(M, N)>0$ и $\xi=\xi(M, N)>0$ как решение системы уравнений

$$
M=\xi^{-1} \sum_{j=1}^{\infty} j q_{j} e^{-\beta j}, \quad N=\xi^{-1} \sum_{j=0}^{\infty} q_{j} e^{-\beta j} .
$$

ПреДЛОЖЕНИЕ 12. Для любых $M, N>0$ система (47) имеет единственное решение $\beta=\beta(M, N)>0, \xi=\xi(M, N)>0$. При $\beta \rightarrow 0$ справедливы асимптотические соотношения

$$
N \asymp \xi^{-1} \beta^{-3}, \quad M \asymp \xi^{-1} \beta^{-4} .
$$

ЗАмЕчаниЕ 13. При $\beta \rightarrow 0$ можно в системе (47) перейти от сумм к интегралам, пользуясь формулами Эйлера-Маклорена. В главном члене при этом получим

$$
N=\xi^{-1} \beta^{-3}(1+O(b)), \quad M=3 \xi^{-1} \beta^{-4}(1+O(b)) .
$$


Отсюда $\beta$ и $\xi$ можно выразить через $M$ и $N$ асимптотическими формулами

$$
\beta=\frac{3 N}{M}+O\left((N / M)^{2}\right), \quad \xi=\frac{M^{3}}{27 N^{4}}(1+O((N / M))) .
$$

В дальнейшем считаем, что $M, N, \beta$ и $\xi$ связаны формулами (47). Кроме того, будем пользоваться обозначениями (11) для суммарных чисел заполнения соответствующих уровней $j$ и положим

$$
\bar{N}_{j}=q_{j} \xi^{-1} e^{-\beta j}, \quad j=0,1,2, \ldots
$$

Наконец, всюду будет предполагаться, что параметры $\beta$ и $\xi$ удовлетворяют соотношению

$$
\xi<\beta^{-3+\varepsilon}
$$

для некоторого произвольного (но фиксированного) $\varepsilon>0$.

3.2. Энтропия и теоремы о концентрации. Обозначим через $\mathscr{N}(M, N)$ общее число упорядоченных выборок, числа заполнения которых удовлетворяют ограничениям (5) и (6), а через

$$
S=\ln \mathscr{N}(M, N)
$$

- энтропию рассматриваемой системы различимых частиц.

Асимптотику энтропии при $N, M \rightarrow \infty$ дает следующая теорема.

Теорема 14. Пусть выполнено условие (51). Тогда при $N, M \rightarrow \infty$ справедлива асимптотическая формула

$$
S=\beta M+N \ln N+N \ln \xi+O(\ln N) .
$$

Пусть, как и ранее, $\psi(x)$ - положительная функция, сколь угодно медленно растущая при $x \rightarrow \infty$.

ТЕОРема 15. Пусть выполнено условие (51). Тогда справедливы оценки

$$
\mathrm{P}\left(\left|\sum_{j=0}^{\infty} j N_{j}-M\right|>\frac{M}{N} \sqrt{N \ln N} \psi(N)\right)=O\left(N^{-k}\right)
$$

для любого $k \in \mathbb{Z}$.

Для кумулятивных вероятностей справедлива следующая

ТЕОРема 16. Пусть выполнено условие (51). Тогда справедливы оценки

$$
\begin{array}{r}
\mathrm{P}\left(\left|\sum_{j=0}^{l} N_{j}-\sum_{j=1}^{l} q_{j} \xi^{-1} e^{-\beta j}\right|>\sqrt{N \ln N} \psi(N)\right)=O\left(N^{-k}\right), \\
\mathrm{P}\left(\left|\sum_{j=1}^{l} j N_{j}-\sum_{j=1}^{l} j q_{j} \xi^{-1} e^{-\beta j}\right|>\frac{M}{N} \sqrt{N \ln N} \psi(N)\right)=O\left(N^{-k}\right)
\end{array}
$$

для любъх $l, k \in \mathbb{Z}_{+}$. 
3.3. Доказательства. Доказательства проводятся таким же методом, как и в случае неразличимых частиц. Основное отличие состоит в методе подсчета вариантов. Если в случае неразличимых частиц имелось взаимно однозначное соответствие между выборками и наборами чисел заполнения, то в случае различимых частиц (т.е. упорядоченных выборок) каждому набору $\left\{N_{j k}\right\}$ чисел заполнения соответствуeT

$$
\frac{N !}{\prod_{j=0}^{\infty} \prod_{k=1}^{q_{j}} N_{j k} !}
$$

различных выборок, где $N=\sum N_{j k}$. Поэтому при подсчете вариантов следует принимать во внимание коэффициенты (56), вводя их в формулы в нужных местах. Перейдем к изложению доказательства, обращая внимание главным образом на отличия от бозевского случая.

3.3.1. Оценка сверху для общего числа вариантов. Обозначим

$$
\mu=\ln \xi, \quad \text { так что } \quad \xi=e^{\mu} .
$$

Пусть $\mathscr{M}$ - множество всех упорядоченных выборок, на котором выполнены условия (5) и (6). Для числа $\mathscr{N}(N, M)$ таких выборок получаем оценку сверху

$$
\mathscr{N}(M, N) \leqslant C \sqrt{N} \exp \{N \ln N+\beta M+\mu N\} .
$$

Действительно,

$$
\begin{aligned}
\mathscr{N}(M, N) & =N ! \sum_{\left\{N_{j k}\right\} \in \mathscr{M}} \frac{1}{\prod_{j=0}^{\infty} \prod_{k=1}^{q_{j}} N_{j k} !} \\
& \leqslant N ! e^{\beta M+\mu N} \sum_{\left\{N_{j k}\right\}} \frac{\exp \left\{-\sum_{j=0}^{\infty} \sum_{k=1}^{q_{j}} N_{j k}(\beta j+\mu)\right\}}{\prod_{j=0}^{\infty} \prod_{k=1}^{q_{j}} N_{j k} !} \\
& =N ! e^{\beta M+\mu N} \prod_{j=0}^{\infty} \prod_{k=1}^{q_{j}} \sum_{N_{j k}=0}^{\infty} \frac{e^{-N_{j k}(\beta j+\mu)}}{N_{j k} !} \\
& =N ! e^{\beta M+\mu N} \prod_{j=0}^{\infty} \prod_{k=1}^{q_{j}} e^{e^{-\beta j-\mu}} \\
& =N ! \exp \left\{\beta M+\mu N+\sum_{j=0}^{\infty} q_{j} e^{-\beta j-\mu}\right\} \\
& =N ! \exp \{\beta M+\mu N+N\} \\
& \leqslant C \sqrt{N} \exp \{N \ln N+\beta M+\mu N\}
\end{aligned}
$$

3.3.2. Оценка числа вариантов с большими уклонениями. Пусть теперь $\mathscr{M}_{\Delta}-$ подмножество в $\mathscr{M}$, состоящее из вариантов, для которых

$$
\left|\sum_{j=0}^{l} \sum_{k=1}^{q_{j}}\left(N_{j k}-\bar{N}_{j k}\right)\right|>\Delta,
$$

где

$$
\bar{N}_{j k}=e^{-\beta j-\mu} \equiv \bar{N}_{j} / q_{j}
$$


(эти числа не зависят от $k$ ). Рассуждая как и выше, для числа $\mathscr{N}(M, N, \Delta)$ выборок во множестве $\mathscr{M}_{\Delta}$ получаем при $0<c<\mu$

$$
\begin{aligned}
\mathscr{N}(M, N, \Delta) \leqslant N ! & \exp \left\{\beta M+\mu N-c \Delta+\sum_{j=l+1}^{\infty} q_{j} e^{-\beta j-\mu}\right\} \\
& \times\left(\exp \left\{\sum_{j=0}^{l}\left(q_{j} e^{-\beta j-\mu+c}-c \bar{N}_{j}\right)\right\}+\exp \left\{\sum_{j=0}^{l}\left(q_{j} e^{-\beta j-\mu-c}+c \bar{N}_{j}\right)\right\}\right),
\end{aligned}
$$

где $\bar{N}_{j}$ задаются формулой (50).

Далее, пользуясь формулой Тейлора с остаточным членом второго порядка, получаем

$$
q_{j} e^{-\beta j-\mu \pm c} \mp c \bar{N}_{j}=q_{j} e^{-\beta j-\mu}\left(e^{ \pm c} \mp c\right)=q_{j} e^{-\beta j-\mu}\left(1+\frac{c^{2}}{2} e^{ \pm \theta c}\right),
$$

где $\theta \equiv \theta(c) \in(0,1)$.

Если $c \leqslant \min \{\mu / 2,1\}$, то получаем неравенство

$$
\sum_{j=0}^{l} q_{j} e^{\beta j+\mu-\theta_{j} c} \leqslant \text { const } e^{-\mu} \beta^{-3} .
$$

Следовательно,

$$
\mathscr{N}(N, M, \Delta) \leqslant C \sqrt{N} \exp \{N \ln N+\beta M+\mu N\} \exp \left\{-c \Delta+K c^{2} e^{-\mu} \beta^{-3}\right\} .
$$

Подставляя сюда

$$
\Delta=\sqrt{N \ln N} \psi(N) \asymp e^{-\mu / 2} \beta^{-3 / 2} \sqrt{\ln N} \psi(N)
$$

И

$$
c=\frac{\beta^{3} e^{\mu} \Delta}{2 K} \asymp \beta^{3 / 2} e^{\mu / 2} \sqrt{\ln N} \psi(N)
$$

(что законно, поскольку при таком выборе $c$ в силу условия (51) неравенство $c \leqslant \mu / 2$ будет заведомо выполнено при достаточно больших $N)$, получим неравенство

$$
\mathscr{N}(N, M, \Delta) \leqslant C_{k} \sqrt{N} \exp \{N \ln N+\beta M+\mu N\} N^{-k}
$$

для любого $k$. Это и есть требуемая оценка сверху для числа вариантов с большими уклонениями.

3.3.3. Оценка снизу для общего числа вариантов. Дадим теперь для $\mathscr{N}(M, N)$ оценку снизу. Как и в бозевском случае, вместо того, что бы оценивать само число $\mathscr{N}(M, N)$, оценим число $\mathscr{N}_{0}(M, N)<\mathscr{N}(M, N)$ выборок, для которых выполнены соотношения (5) и (6), причем последнее также обращается в равенство:

$$
\sum_{j=0}^{\infty} \sum_{k=1}^{q_{j}} N_{j k}=N, \quad \sum_{j=0}^{\infty} \sum_{k=1}^{q_{j}} j N_{j k}=M
$$


Множество наборов чисел заполнения, удовлетворяющих (67), обозначим через $\mathscr{M}_{0}$. Тогда

$$
\begin{aligned}
\mathscr{N}_{0}(M, N) & =\sum_{\left\{N_{j k}\right\} \in \mathscr{M}_{0}} \frac{N !}{\prod_{j=0}^{\infty} \prod_{k=1}^{q_{j}} N_{j k} !} \\
& =N ! \sum_{\left\{N_{j k}\right\}} \frac{\delta\left(N, \sum_{j=0}^{\infty} \sum_{k=1}^{q_{j}} N_{j k}\right) \delta\left(M, \sum_{j=0}^{\infty} \sum_{k=1}^{q_{j}} j N_{j k}\right)}{\prod_{j=0}^{\infty} \prod_{k=1}^{q_{j}} N_{j k} !} .
\end{aligned}
$$

Здесь сумма во второй строчке берется по всем финитным наборам неотрицательных чисел заполнения, а

$$
\delta(m, n) \equiv \delta_{m n}
$$

- символ Кронекера.

Подставим интегральное представление

$$
\delta_{m n}=\frac{s}{2 \pi} \int_{-\pi / s}^{\pi / s} e^{(i s x+\omega)(m-n)} d x
$$

символа Кронекера (где $s$ и $\omega$ - произвольные ненулевые вещественные числа) в (68), выбирая для первого сомножителя $s=1$ и $\omega=\mu$, а для второго $-s=\omega=\beta$. Тогда для $\mathscr{N}_{0}(M, N)$ получим интегральное представление

$$
\mathscr{N}_{0}(M, N)=\frac{\beta N ! e^{\beta M+\mu N}}{4 \pi^{2}} \int_{-\pi / \beta}^{\pi / \beta}\left(\int_{-\pi}^{\pi} e^{\Lambda \Phi(\varphi, \psi)} d \psi\right) d \varphi,
$$

где

$$
\begin{gathered}
\Lambda=\beta^{-3} e^{-\mu} \asymp N \\
\Phi(\varphi, \psi)=i \beta^{4} e^{\mu} M \varphi+i \beta^{3} e^{\mu} N \psi+\beta^{3} \sum_{j=0}^{\infty} q_{j} e^{-\beta j-i(\psi+\beta j \varphi)} .
\end{gathered}
$$

Действительно, указанная подстановка дает

$$
\begin{aligned}
& \mathscr{N}_{0}(M, N)=\frac{\beta N ! e^{\beta M+\mu N}}{4 \pi^{2}} \int_{-\pi / \beta}^{\pi / \beta}\left(\int_{-\pi}^{\pi} \sum_{\left\{N_{j k}\right\}} \frac{e^{i \beta \varphi M+i \psi N}}{\prod_{j=0}^{\infty} \prod_{k=1}^{q_{j}} N_{j k} !}\right. \\
& \left.\times \exp \left\{-\sum_{j=0}^{\infty} \sum_{k=1}^{q_{j}} N_{j k}(\beta j+\mu+i \beta j \varphi+i \psi)\right\} d \psi\right) d \varphi \\
& =\frac{\beta N ! e^{\beta M+\mu N}}{4 \pi^{2}} \int_{-\pi / \beta}^{\pi / \beta}\left(\int_{-\pi}^{\pi} e^{i \beta \varphi M+i \psi N}\right. \\
& \left.\times \prod_{j=0}^{\infty} \prod_{k=1}^{q_{j}} \sum_{N_{j k}=0}^{\infty} \frac{e^{-N_{j k}(\beta j+\mu+i \beta j \varphi+i \psi)}}{N_{j k} !} d \psi\right) d \varphi \\
& =\frac{\beta N ! e^{\beta M+\mu N}}{4 \pi^{2}} \int_{-\pi / \beta}^{\pi / \beta}\left(\int_{-\pi}^{\pi} e^{i \beta \varphi M+i \psi N}\right. \\
& \left.\times \prod_{j=0}^{\infty} \prod_{k=1}^{q_{j}} \exp \left\{e^{-(\beta j+\mu+i \beta j \varphi+i \psi)}\right\} d \psi\right) d \varphi
\end{aligned}
$$




$$
\begin{aligned}
=\frac{\beta N ! e^{\beta M+\mu N}}{4 \pi^{2}} \int_{-\pi / \beta}^{\pi / \beta}\left(\int_{-\pi}^{\pi} \exp \{i \beta \varphi M+i \psi N\right. \\
\left.\left.+\sum_{j=0}^{\infty} q_{j} e^{-(\beta j+\mu+i \beta j \varphi+i \psi)}\right\} d \psi\right) d \varphi .
\end{aligned}
$$

Лемма 17. Задаваемая формулой (71) фазовая функция $\Phi(\varphi, \psi)$ обладает следующими свойствами:

1) все ее производные равномерно ограничены при значениях параметров $\beta$ и удовлетворяющих неравенству (51);

2) фазовая функиия имеет стационарную точку $\varphi=0 \bmod 2 \pi / \beta, \psi=0 \bmod 2 \pi$;

3) матрица $\Phi^{\prime \prime}(0,0)$ вторьх производнъх фазовой функиии в стационарной точке невырождена и равномерно по параметрам $\beta$ и $\xi$, удовлетворяющим неравенству (51), отрицательно определена;

4) мнимая часть фазовой функции в стационарной точке равна нулю, а ее вещественная часть достигает там абсолютного максимума, причем для любого $\gamma>0$ существует такое $\delta>0$, не зависящее от параметров $\beta$ и $\xi$, удовлетворяющих неравенству (51), что

$$
\operatorname{Re} \Phi(\varphi, \psi)<\operatorname{Re} \Phi(0,0)-\delta \quad n p u \quad \operatorname{dist}((\varphi, \psi),(0,0))>\gamma
$$

ДокАЗАтЕЛьСтво. 1) Ограниченность производных фазовой функции доказывается непосредственной выкладкой.

2) Чтобы проверить, что точка $(0,0)$ - стационарная точка фазовой функции, вычислим ее первые производные:

$$
\begin{aligned}
& \frac{\partial \Phi}{\partial \varphi}=i \beta^{4} e^{\mu}\left[M-e^{-\mu} \sum_{j=0}^{\infty} j q_{j} e^{-\beta j-i(\psi+\beta j \varphi)}\right], \\
& \frac{\partial \Phi}{\partial \psi}=i \beta^{3} e^{\mu}\left[N-e^{-\mu} \sum_{j=0}^{\infty} q_{j} e^{-\beta j-i(\psi+\beta j \varphi)}\right] .
\end{aligned}
$$

При $\varphi=\psi=0$ обе производные обращаются в нуль в силу определения параметров $\beta$ и $\xi=e^{\mu}$.

3) Матрица $\Phi^{\prime \prime}(0,0)$ имеет вид

$$
\Phi^{\prime \prime}(0,0)=-\sum_{j=0}^{\infty} \beta^{3} q_{j} e^{-\beta j}\left(\begin{array}{cc}
1 & \beta j \\
\beta j & \beta^{2} j^{2}
\end{array}\right) .
$$

Оценим эту матрицу, рассматривая ее как матрицу квадратичной формы, следующим образом:

$$
\Phi^{\prime \prime}(0,0) \leqslant-\sum_{j=\left[x_{1} / \beta\right]}^{\left[x_{2} / \beta\right]} \beta^{3} q_{j} e^{-\beta j}\left(\begin{array}{cc}
1 & \beta j \\
\beta j & \beta^{2} j^{2}
\end{array}\right),
$$

где $x_{2}>x_{1}>0$ - произвольные фиксированные числа. Дальнейшее рассуждение ничем не отличается от (38). 
4) Из формулы $(71)$ для $\Phi(\varphi, \psi)$ получаем

$$
\operatorname{Re} \Phi(0,0)-\operatorname{Re} \Phi(\varphi, \psi)=\beta^{3} \sum_{j=0}^{\infty} q_{j} e^{-\beta j}(1-\cos (\psi+\beta j \varphi)) .
$$

Все слагаемые в правой части здесь неотрицательны. Поэтому, опуская часть из них и оценивая коэффициенты $\beta^{2} q_{j} e^{-\beta j}$ для оставшихся слагаемых константой, получаем

$$
\begin{aligned}
\operatorname{Re} \Phi(0,0)-\operatorname{Re} \Phi(\varphi, \psi) & \geqslant \operatorname{const} \beta \sum_{j=\left[x_{1} / \beta\right]}^{\left[x_{2} / \beta\right]}(1-\cos (j \varphi+\psi)) \\
& \geqslant \operatorname{const}\left(x_{2}-x_{1}-\beta-\beta\left|\sin \frac{\beta \varphi}{2}\right|^{-1}\right),
\end{aligned}
$$

откуда, подбирая $x_{1}$ и $x_{2}$ подходящим образом, получаем требуемое утверждение. Лемма доказана.

Доказанная только что лемма позволяет вычислить интеграл (69), следуя рассуждениям, проведенным в предыдущем параграфе, и получить оценку снизу для числа упорядоченных выборок в виде

$$
\mathscr{N}(N, M) \geqslant C \beta \Lambda^{-1} \sqrt{N} \exp \{N \ln N+\beta M+\mu N\} .
$$

3.3.4. Вычисление энтропии и доказательство теорем о концентрации. Теперь несложно доказать теоремы 8-10, используя полученные выше оценки. Рассуждение дословно повторяет то, что было использовано в бозевском случае.

\section{4. Распределение Бозе-Эйнштейна в случае относительно малого числа частиц}

Вернемся теперь к рассмотрению системы бозе-частиц (5), (6) и выясним, как ведет себя распределение, описанное в теореме 10 , когда $M$ и $N$ стремятся к бесконечности, но при этом $N$ возрастает сравнительно медленно (точную формулировку см. ниже).

Напомним [1], что если в системе бозе-частиц с целочисленными уровнями энергии задано только ограничение $M$ сверху на суммарную энергию, а число частиц произвольно ${ }^{2}$, то для большинства выборок число частиц будет мало отличаться от некоторого значения $N_{0}$, которое определяется по $M$. Соответствующие формулы были выписаны в [1] и для интересующего нас случая $d=3$ имеют следующий вид. Пусть $b=b(M)-($ единственный $)$ положительный корень уравнения

$$
M=3 \zeta(4) b^{-4}+3 \zeta(3) b^{-3} \equiv \frac{\pi^{4}}{30} b^{-4}+3,606 \ldots b^{-3}
$$

относительно переменной $b$, где $\zeta(x)$ - дзета-функция Римана. Тогда

$$
N_{0}=\zeta(3) b^{-3}+\frac{3}{2} \zeta(2) b^{-2} \equiv 1,202 \ldots b^{-3}+\frac{\pi^{2}}{4} b^{-2} .
$$

\footnotetext{
${ }^{2}$ При этом, конечно, надо считать, что уровни энергии начинаются не с нуля, а с единицы; см. замену переменных (7) в замечании 4.
} 
Очевидно,

$$
N_{0} \asymp b^{-3} \asymp M^{3 / 4}, \quad \frac{N_{0}}{M} \asymp b .
$$

Пусть теперь мы не только задали $M$, но и зафиксировали число частиц $N$. Что будет в том случае, если $N$ отличается от $N_{0}$ ? В работе [2] был исследован случай, когда $N \gg N_{0}$ и показано, что в этом случае образуется конденсат - «лишние» частицы скапливаются на нижнем уровне. Выясним, что будет происходить в противоположном случае, когда $N_{0} \gg N$ (точнее говоря, $N$ стремится к бесконечности вместе с $M$, но гораздо медленнее, чем $N_{0}$ ). Оказывается, что при этом условии частицы распределены по «гиббсовскому» закону.

ТеОРема 18. Пусть $M, N \rightarrow \infty$ таким образом, что выполнено условие (51) и

$$
N / N_{0} \leqslant C b^{1 / 3}, \quad N_{0} \geqslant C b^{\delta}
$$

для некоторых постоянных $C, \delta>0$. Тогда большинство наборов $\left\{N_{j}\right\}$ концентрируется вблизи «гиббсовского» распределения

$$
\bar{N}_{j}=\xi^{-1} q_{j} e^{-\beta j}
$$

где параметры $\xi$ и $\beta$ определяются из системъь ${ }^{3}$ (8). Более точно,

$$
\begin{array}{r}
\mathrm{P}\left(\left|\sum_{j=0}^{s} \sum_{k=1}^{q_{j}}\left(N_{j}-\bar{N}_{j}\right)\right|>\sqrt{N \ln N} \psi(N)\right)=O\left(N^{-k}\right), \\
\mathrm{P}\left(\left|\sum_{j=0}^{s} \sum_{k=1}^{q_{j}} j\left(N_{j}-\bar{N}_{j}\right)\right|>\frac{M}{N} \sqrt{N \ln N} \psi(N)\right)=O\left(N^{-k}\right)
\end{array}
$$

для любых $s, k \in \mathbb{Z}$.

ЗАмЕчАНИЕ 19. Мы поставили слово «гиббсовское» в кавычки по следующим причинам. Хотя и формула для распределения, вблизи которого концентрируется большинство вариантов, и оценка вероятности больших уклонений от этого распределения идентичны тем, что были получены в разделе 3 для гиббсовского распределения, число вариантов (и, следовательно, энтропия системы) соответствуют не гиббсовскому (теорема 14), а бозевскому случаю (теорема 8).

ДокАзАтЕЛЬСтво. Наряду с распределением (80) рассмотрим бозевское распределение

$$
\bar{n}_{j}=\frac{q_{j}}{\xi e^{\beta j}-1}
$$

с теми же параметрами $(\beta, \xi)$. Очевидно,

$$
\bar{n}_{j}>\bar{N}_{j}, \quad j=0,1,2, \ldots
$$

Теорема 10 утверждает, что соотношения (81), (82) выполнены с заменой $\bar{N}_{j}$ на $\bar{n}_{j}$. Поэтому для того, чтобы доказать требуемое утверждение, достаточно показать,

\footnotetext{
${ }^{3}$ Можно определять их и из системы (47); при этом утверждение теоремы останется верным.
} 
что при наших предположениях справедлива оценка ${ }^{4}$

$$
\sum_{j=0}^{\infty}\left(\bar{n}_{j}-\bar{N}_{j}\right)=O(\sqrt{N})=O\left(\xi^{-1 / 2} \beta^{-3 / 2}\right) .
$$

Имеем

$$
\bar{n}_{j}-\bar{N}_{j}=\frac{q_{j}}{\xi e^{\beta j}-1}-\frac{q_{j}}{\xi e^{\beta j}} \asymp q_{j} \xi^{-2} e^{-2 \beta j}
$$

(последнее соотношение справедливо в силу того, что $\xi$ велико, что в свою очередь вытекает из малости отношения $\left.N / N_{0}\right)$. Поэтому

$$
\sum_{j=0}^{\infty}\left(\bar{n}_{j}-\bar{N}_{j}\right) \asymp \xi^{-2} \sum_{j=0}^{\infty} q_{j} e^{-2 \beta j} \asymp \xi^{-2} \beta^{-3} .
$$

Для того чтобы последнее выражение было $O(\sqrt{N})$, достаточно, чтобы

$$
\xi^{-2} \beta^{-3} \leqslant C \xi^{-1 / 2} \beta^{-3 / 2},
$$

или

$$
\xi \geqslant C \beta^{-1} \text {. }
$$

Имеют место соотношения

$$
\frac{M}{N_{0}} \asymp b^{-1}, \quad \frac{M}{N} \asymp \beta^{-1},
$$

откуда

$$
\frac{N}{N_{0}}=\frac{\beta}{b}
$$

Далее,

$$
\frac{M^{3}}{N_{0}^{4}} \asymp 1, \quad \frac{M^{3}}{N^{4}} \asymp \xi \geqslant C \beta^{-1},
$$

откуда получаем неравенство

$$
\left(\frac{N}{N_{0}}\right)^{4} \leqslant C_{1} \beta
$$

Итак,

$$
\frac{\beta^{4}}{b^{4}} \leqslant C_{1} \beta
$$

или

$$
\beta \leqslant C_{2} b^{4 / 3}
$$

или, окончательно,

$$
\frac{N}{N_{0}} \leqslant C b^{1 / 3}
$$

что и завершает доказательство.

\footnotetext{
4 Достаточно и более слабой оценки с дополнительным множителем $\sqrt{\ln N} \psi(N)$, так что и утверждение теоремы можно соответственно уточнить, чего мы не стали делать во избежание громоздкости.
} 


\section{СПИСОК ЦИТИРОВАННОЙ ЛИТЕРАТУРЫ}

[1] В. П. Маслов, В.Е. Назайкинский, "О распределении целочисленных случайных величин, связанных одним линейным неравенством. I", Матем. заметки, 83:2 (2008), $232-263$.

[2] В.П. Маслов, В.Е. Назайкинский, "О распределении целочисленных случайных величин, связанных одним линейным неравенством. II", Матем. заметки, 83:3 (2008), 381-401.

[3] И. С. Градштейн, И. М. Рыжик, Таблицы интегралов, сумм, рядов и произведений, ГИФМЛ, М., 1963.

[4] А.Н. Ширяев, Вероятность, кн. 1: Элементарная теория вероятностей. Математические основания. Предельные теоремы, МЦНМО, М., 2004.

В. П. Маслов

Поступило

Московский государственный университет

20.03.2008

им. М. В. Ломоносова

E-mail: v.p.maslov@mail.ru

В. Е. Назайкинский

Институт проблем механики РАН

E-mail: nazaikinskii@yandex.ru 